\title{
An Abstract Model for Service Compositions Based on Agents ${ }^{\star}$
}

\author{
Jinkui Xie and Linpeng Huang \\ Dept. of Computer Science and Engineering, Shanghai Jiao Tong University, \\ Shanghai 200030, P.R. China \\ $\{j k x i e$, huang-lp\}@cs.sjtu.edu.cn
}

\begin{abstract}
This paper presents an abstract model for service compositions based on agents. The model is based on distributed Abstract State Machine and tallies with Universal Plug and Play (UPnP) architecture standard. In the abstract model, the composition of services can be viewed as a union of agents. This model can be used to depict a number of Web Service applications, and as an instance, the paper presents a BPEL Abstract Machine.
\end{abstract}

\section{Introduction}

This paper presents an abstract model for service compositions. The abstract model constructs the whole service compositions system as a collection of communicating agent subsystems. Each subsystem's communication structure is analogous in the essence. The model is open and has a Universal Plug and Play (UPnP) architecture [7]. We set up the model with distributed Abstract State Machine, and give some descriptions to BPEL (Business Process Execution Language for Web Services) [6].

\section{$2 \quad$ Abstract State Machines}

We first define the notion of Abstract State Machines. For more details, we refer to the Lipari-Guide [4] and the ASM 1997 Guide [5]. An Abstract State Machine (ASM) is a tuple $\left(\Sigma, \Phi_{\text {Init }}\right.$, Trans $)$ where $\Sigma$ is a signature, $\Phi_{\text {Init }}$ is a set of $\Sigma$ formulas (the initial conditions), and Trans is a finite set of transition rules. The set of states is the set $\operatorname{Alg}(\Sigma)$ of $\Sigma$-algebras; $\llbracket \cdot \rrbracket_{q}$ denotes the interpretation function of symbols of $\Sigma$ in $\Sigma$-algebra $q$. A state $q$ is initial iff $q$ is a model of $\Phi_{\text {Init }}$ in the sense of logic, denoted as $q \models \Phi_{\text {Init }}$. In this article, we use ordersorted partial $\Sigma$-algebras.

For the service compositions' purpose, we introduce A distributed ASM (DASM) involves a collection of agents [3]. Agents are represented in global states as well. They are elements of a dynamically universe Agent that may

\footnotetext{
* This work is supported by "SEC E-institute: Shanghai High Institutions Grid" project 200308 .
} 
grow and shrink. Intuitively, a run can be seen as the common part of histories of the same computation recorded by various observers.

\section{Service Compositions Based on Agents}

Research in the Web Service model is a hot area. In our abstract model, Web Service infrastructure can be divided into three layers: the Service Requestor Layer, the Middle Agent Layer, and the Service Layer [1]. Moreover, in this system, the Agent category has three subcategories: the Service Requestor Agent (SRA), the Service Provide Agent (SPA), and the Coordination Agent (COA). Among these subcategories, the agents are flexible and can transform their roles in the Web Service context, so the whole composition system is a Universal Plug and Play (UPnP) architecture [7].

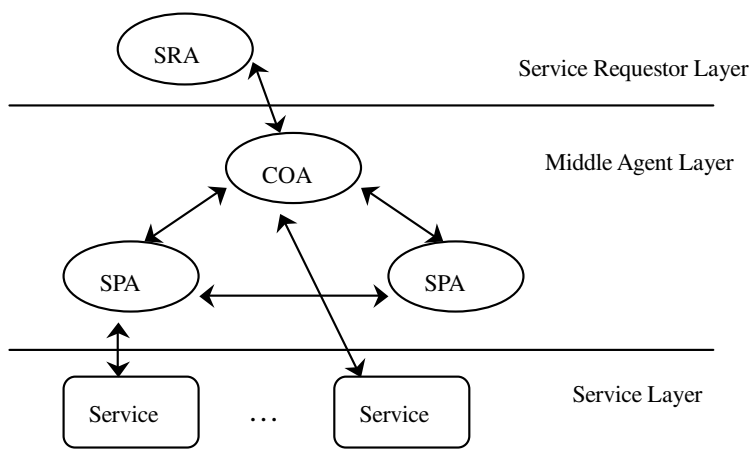

Fig. 1. Agent-based service compositions framework

The agent-based service compositions framework is presented on Fig. 1. In the model, there are three basic operations: (1) agent 1 ○ agent 2 : it represents a composite service that performs the service agent $t_{1}$ followed by the service agent $_{2}$, i.e., $\circ$ is an operator of sequence. (2) agent $t_{1}+$ agent $_{2}$ : it represents a composite service that behaves as either service agent $t_{1}$ or service agent . Once . one of them executes its operation the other service is discarded, i.e., + is an operator of choice. (3) agent $\left\|_{1}\right\|$ agent $_{2}$ : it represents a composite service that performs the services agent ${ }_{1}$ and agent $_{2}$ independently, i.e., $\|$ is an operator of concurrence.

\section{Service Compositions Abstract Machine}

Based on DASM, we can present a BPEL Abstract Machine. The BPEL (Business Process Execution Language for Web Services) builds on top of WSDL (and indirectly also on SOAP) effectively introducing a stateful interaction model that allows to exchange sequences of messages between business partners (i.e. Web 


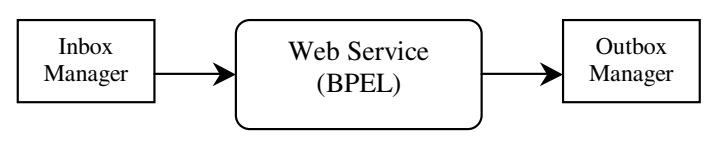

Fig. 2. High-level structure of the BPEL model

services) [6]. A BPEL document abstractly defines a Web service consisting of a collection of business process instances. A process instance maintains a continuous interaction with the external world (i.e., the communication network) through two interface components, called inbox manager and outbox manager [2], as shown in Fig. 2.

\section{domain AGENT $\equiv$ INBOX_MANAGER $\cup$ OUTBOX_MANAGER $\cup$ PROCESS $\cup$ ACTIVITY_AGENT $\cup$ HANDLER_AGENT}

In the initial DASM state, there are only three DASM agents: the inbox manager, the outbox manager and a dummy process.

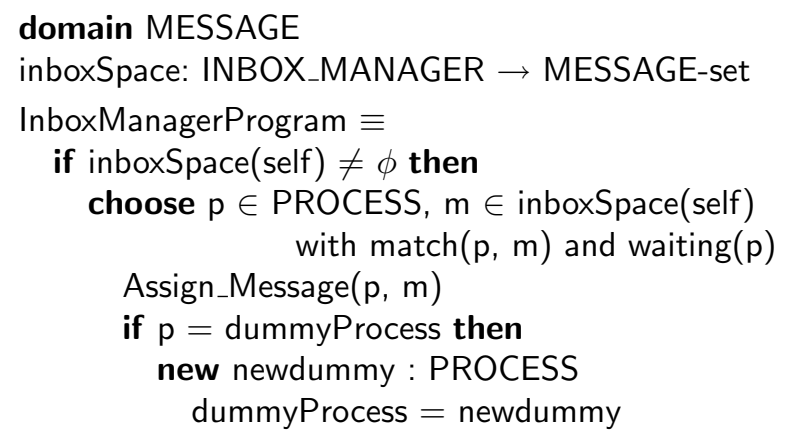

In general, a BPEL program combines two different types of activities: basic activities and structured activities.

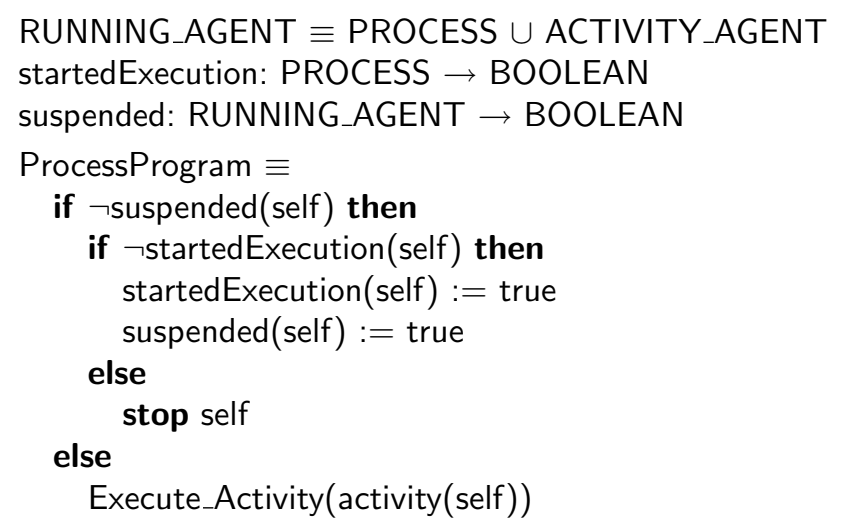

ReceiveMode: RUNNING_AGENT $\rightarrow$ BOOLEAN

waitingForMessage: PROCESS $\rightarrow\langle$ RUNNING_AGENT, ACTIVITY $\rangle$-set

Execute_Receive(activity: RECEIVE) $\equiv$

let inputDescriptor $=\langle$ self, activity

if $\neg$ receiveMode(self) then 


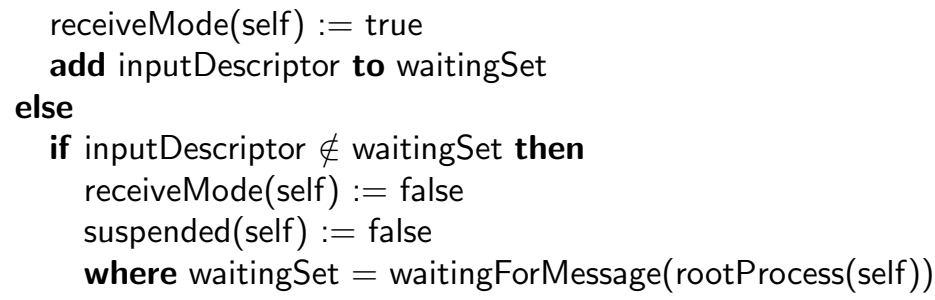

A basic agent executes a single activity. Thus, its program is very similar to a process agent.

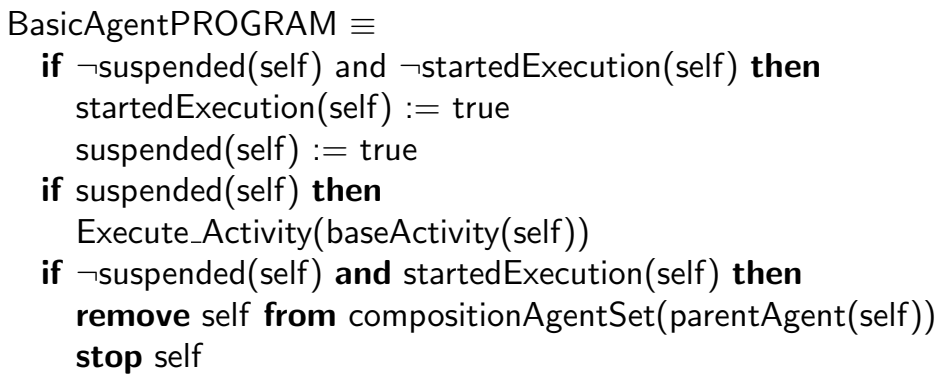

\section{Conclusions}

In this paper, we introduce a distributed ASM (DASM) involves a collection of agents, then provide an abstract service architecture based on agents, which is open and universal. As an application, the paper presents a BPEL Abstract Machine. The proposed hierarchical abstract framework enhances the intelligibility and integrality of the business process. The future work of the model will focus on the complicated business interactions in the service.

\section{References}

1. V. Ermolayev, N. Keberle. Towards a Framework for Agent-Enabled Semantic Web Service Composition, International Journal of Web Service Research, Volume X, No. X, 2004.

2. R. Farahbod, U. Glässer and M. Vajihollahi. Specification and Validation of the Business Process Execution Language for Web Services, ASM 2004, LNCS 3052.

3. U. Glässer, Y. Gurevich and M. Veanes. An Abstract Communication Model, Microsoft Research Technical Report MSR-TR-2002-55, May 2002.

4. Y. Gurevich. Evolving algebras 1993: Lipari guide. In E. Börger, editor, Specification and Validation Methods, pages 9-36. Oxford University Press, 1995.

5. Y. Gurevich. May 1997 draft of the ASM guide. Technical Report CSE-TR-336-97, Univer-sity of Michigan EECS Department, 1997.

6. Specification: Business Process Execution Language for Web Services, Version 1.1, 2003. http://www-106.ibm.com/developerworks/library/ws-bpel/

7. UPnP Device Architecture V1.0. Microsoft Universal Plug and Play Summit, Seattle 2000, Microsoft Corporation, Jan. 2000. 